\title{
Sexual selection and genital evolution
}

\author{
David J. Hosken ${ }^{1}$ and Paula Stockley ${ }^{2}$ \\ ${ }^{1}$ Zoologisches Museum, University of Zurich-Irchel, Winterthurerstrasse 190, CH-8057 Zurich, Switzerland \\ ${ }^{2}$ Animal Behaviour Group, Faculty of Veterinary Science, University of Liverpool, Leahurst, Neston, South Wirral CH647TE UK
}

\begin{abstract}
Genitalia are conspicuously variable, even in closely related taxa that are otherwise morphologically very similar. Explaining genital diversity is a longstanding problem that is attracting renewed interest from evolutionary biologists. New studies provide ever more compelling evidence that sexual selection is important in driving genital divergence. Importantly, several studies now link variation in genital morphology directly to male fertilization success, and modern comparative techniques have confirmed predicted associations between genital complexity and mating patterns across species. There is also evidence that male and female genitalia can coevolve antagonistically. Determining mechanisms of genital evolution is an important challenge if we are to resolve current debate concerning the relative significance of mate choice benefits and sexual conflict in sexual selection.
\end{abstract}

Extreme variation in male genital morphology is a conspicuous and comprehensive trend across animals with internal fertilization to the extent that even closely related species with similar general morphology often have strikingly different genitalia (Figure 1). Explaining genital diversity has been a longstanding problem for evolutionary biologists, and the selective pressure(s) responsible for this bewildering array has been the subject of ongoing debate [1-3]. Complicated and divergent morphology is unlikely to have arisen purely for the relatively simple function of sperm transfer. So why the enormous variation (Box 1)?

Before the recent expansion of studies investigating the role of sexual selection in genital evolution, two general non-sexual selection explanations had been proposed (Box 2), neither of which are currently well supported (reviewed in $[1,2,4]$ ). By contrast, evidence is accumulating for a role of sexual selection in genital evolution. The idea that sexual selection influences genital evolution has been extensively developed by Eberhard in the context of postcopulatory sexual selection [1,4-6]. In particular, he has argued that genital divergence is often driven by cryptic female choice, broadly defined as any postcoupling process or structure controlled by females that biases paternity towards males having a certain phenotype when females have mated with more than one male [7]. Eberhard further argues that the female benefit for this choice is most often Fisherian (Box 3). This has generated debate, however, because it can be difficult to distinguish mechanisms of cryptic female choice for sexy sons from good genes, or from other potential selection pressures involved in postcopulatory sexual selection, such as sperm competition and sexual conflict. The evolutionary effects of purely Fisherian benefits are also unclear (Box 3). Moreover, sperm competition probably played at least some role in the initial evolution of male genitalia from a primordial state where gametes were released into the environment [8]. Hence, although a new generation of studies is consistent in supporting Eberhard's hypothesis that sexual selection is the primary force driving genital divergence, the exact selection mechanisms involved are less clear. Here, we discuss recent advances in our understanding of genital evolution, and the challenge of determining the relative importance of various mechanisms of sexual selection in the evolution of genital diversity.

\section{Genital form and fertilization success}

Early evidence for sexual selection driving genital diversity was based partly on observations of genital movement during copulation or postcopulatory behaviours that apparently influence patterns of sperm storage $[1,4,9]$. Observations such as these led Eberhard [1,4] to suggest that genital complexity was driven by Fisherian sexual selection (Box 3), with females cryptically biasing paternity in favour of males that were better copulatory stimulators, and enjoying increased fitness benefits via the inherited ability of their sons to stimulate females (Box 3). However, in most instances, direct evidence that genital variation influenced fertilization success (or some other cryptic process) was not available.

This paucity has now been partly redressed by recent studies of insects, which provide convincing evidence for associations between genital morphology and fertilization success. The first of these, by Arnqvist and Danielsson, looked at fertilization success and the shape of male genital sclerites (plates) in the water strider Gerris lateralis when females mated with two males [3]. After controlling for body size, it was found that sclerite shape influenced fertilization success: males that had stout ventral sclerites and narrow and inwardly curved dorsal sclerites sired significantly more offspring. No other characters measured explained

\section{Box 1. The big picture}

- Genitalia evolve rapidly and divergently, for which sexual selection appears responsible.

- The exact sexual selection mechanism involved is not clear.

- Finding the precise mechanism will help determine the relative importance of conflicts and benefits in sexual selection. 


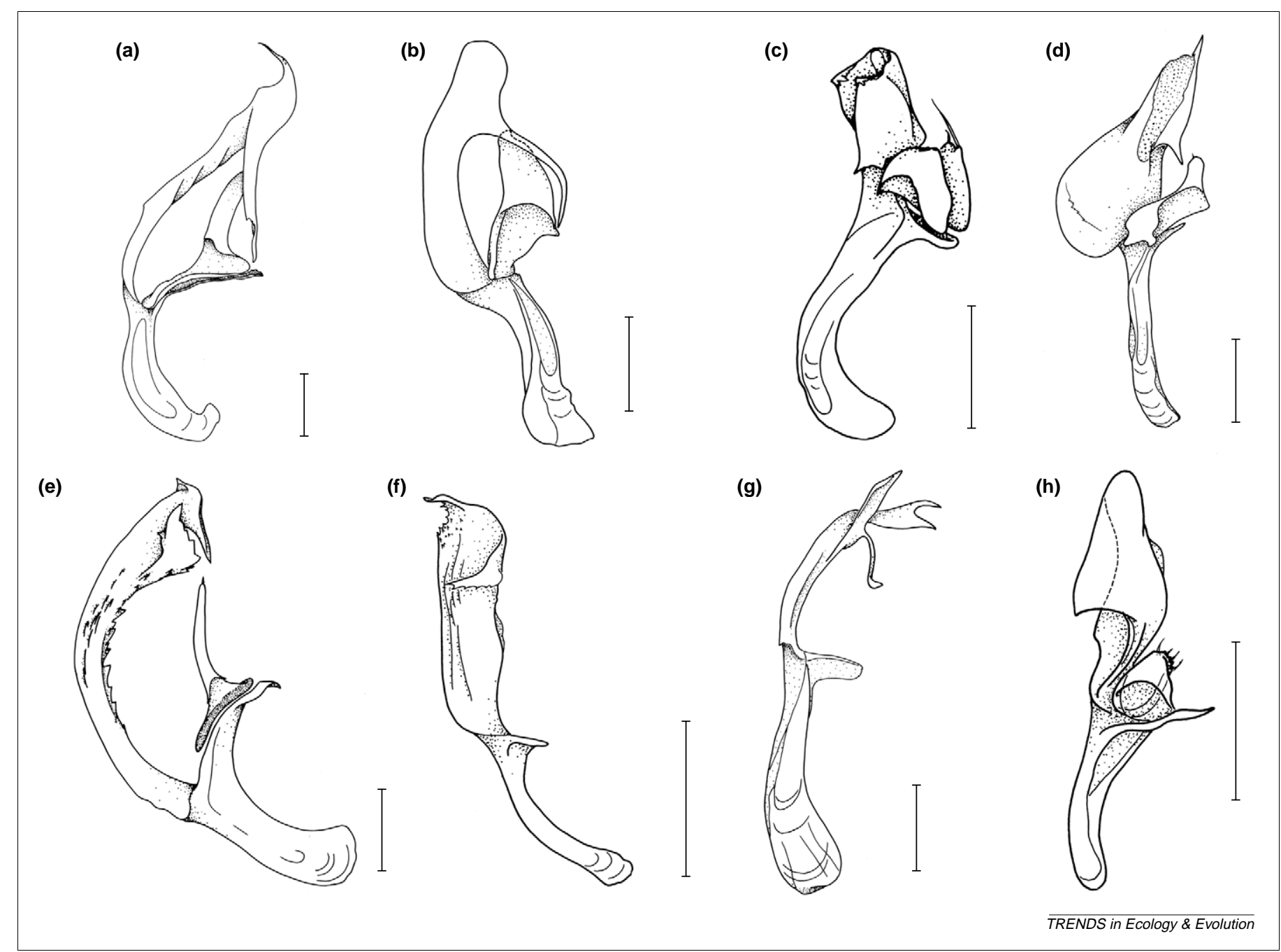

Figure 1. An example of genital variability within the genus Drosophila. Shown in ventral view are the aedeagus, aedeagal apodeme and paraphyses of $D$. guacamaya (a), D. papei (b), D. periquito (c), D. ranchograndensis (d), D. tschirnhausi (e), D. merzi (f), D. pittieri (no aedeagus shown) (g) and D. luiserrai (h). Reproduced with permission from $[72,73]$. Scale bars $(100 \mu \mathrm{m}$.

any variation in paternity, leading the authors to suggest that genital morphology is a largely overlooked source of the residual variance typically seen in sperm competition studies [3]. Similar results were also found in a second water strider Gerris lacustris [10]. A third study, of the

\section{Box 2. Mechanisms of genital evolution not involving sexual selection}

The two major non-sexual selection mechanisms previously thought to be responsible for genital evolution are the lock-and-key and the pleiotropy hypotheses $[1,2,61]$. The lock-and-key hypothesis proposes that genital divergence occurs as a result of hybridization avoidance, with only males of the correct species able to supply the right key to fit the female lock. This idea was widely supported by taxonomists, but is not consistent with evidence based on the morphology of the female reproductive tract, genital associations during copula, intraspecific variation in genital form, or geographical variation in genitalia [1,4]. The pleiotropy (neutral evolution) hypothesis [61] suggests that genitals diverge via pleiotropic effects of selection on other traits. Although this idea is supported by some evidence that pleiotropic gene effects can influence genital organs [51], its general applicability is questionable, in part, because it is unclear why pleiotropy should so disproportionately influence genital organs. dung beetle Onthophagus taurus, also used a two-male sperm competition experiment, and four out of the five measured genital components were found to influence fertilization success [11]. In both the beetle [11] and Arnqvist and Danielsson's [3] water strider study, different components of the aedeagus (the insect intromittent organ) were associated with sperm offence (the ability to gain precedence against rival sperm) and sperm defence (the ability to resist rival sperm precedence attempts). This independence of characters influencing the offensive and defensive performance of males probably contributes to genital complexity [11]. Direct evidence for an association between male fertilization success and genital form has also been found in the fly Dryomyza anilis [12], and work on tortoise beetles documents variation in paternity associated with the length of the genital flagellum, as well as rapid divergence in genital length across species [13].

Less direct methods have also been used when assessing the likelihood that sexual selection has shaped genital form. Tadler [14] used seedbug Lygaeus simulans sisters assigned to random males to look at associations between aedeagus length and successful sperm transfer. Seedbugs 


\section{Box 3. Sexual selection mechanisms and genital evolution}

Four main sexual selection mechanisms have been implicated in genital evolution: sexy sons (also known as Runaway or Fisherian selection), good genes, sperm competition and sexual conflict [20]. According to both the sexy sons and good genes hypotheses, genital divergence is driven by cryptic female choice for males with genitalia that best stimulate them during copulation. Fitness benefits associated with the sexy sons mechanism involve only the inherited mate-attracting potential or, in the present context, female stimulation potential, of the sons of the female, whereas the benefits of good genes additionally include increased offspring viability. The relative importance of these potential selection pressures is the subject of much ongoing theoretical debate [62-66].

Sperm competition (when the ejaculates of two or more males compete to fertilize an ovum) [8] could also cause genital divergence. For example, in odonates [18], selection has favoured males with genitalia that remove rival sperm from the female (so, strictly speaking, selection is by sperm competition avoidance), hence male-male competition rather than female choice per se might be the primary force acting on the odonate aedeagus. However, females could potentially still benefit indirectly through the sperm removal ability of their sons.

Similarly, conflict between the sexes over fertilization decisions might also be involved in the evolution of genitalia [1,33]. Sexual conflict, (i.e. evolutionary conflict between the sexes), will occur whenever there is not strict genetic monogamy and reproduction is costly $[33,34,67]$. Given the widespread occurrence of both these conditions, sexual conflict is rife and can favour traits that increase the fitness of one sex even when it is costly to the other. Hence, elaborate genitals might be the result of an evolutionary arms race between males and females in the struggle for control over reproduction [33], although recent evidence suggests widespread genital divergence is not the result of conflict over mating per se [31]. Sensory exploitation [68], also implicated in genital evolution, could represent the initial evolutionary step in any of the above mechanisms. Distinguishing which mechanisms are more important in the evolution of sexually selected traits, including genitalia, is a key challenge for researchers in this field.

are promiscuous and, for males to transfer sperm successfully, they must insert part of their aedeagus through a valve between the sperm store of the female and the long convoluted duct that leads to it. Sperm counts provided evidence of stabilizing selection (Box 4) on the length of the aedeagal process, and it was suggested that cryptic female choice was the mechanism involved. However, a second study found no evidence for selection on this trait [15].

In the damselfly Calopteryx haemorrhoidalis, the aedeagus distorts a cuticular plate in the female reproductive tract, resulting in ejection of previously stored sperm from the spermatheca of the female [16]. It was proposed that males provide similar stimulation during copulation to that caused by eggs moving down the female reproductive tract, mobilizing stored sperm. Subsequent work has shown that females of more basal species within this group are equally stimulated by the aedeagus of male $C$. haemorrhoidalis, but conspecific males do not incite a sperm ejection response in these taxa [17], further suggesting that sensory exploitation underlies this process (Box 3). This is just one example of the odonate aedeagus being involved in sperm removal, and hence being subject to sexual selection $([18,19]$ reviewed in $[20])$. Sperm removal provides a

\section{Box 4. Selection, allometry and variation}

Selection acts in three general ways: directional, disruptive or stabilizing. Directional selection favours phenotypes with trait values at one end of the trait distribution. For example, if larger bodied individuals have higher fitness, then size can be said to be subject to directional selection. This type of selection is thought to be responsible for the evolution of many sexually selected display traits, such as the peacock's tail. Disruptive selection occurs when the intermediate phenotype is selected against; that is, when more extreme phenotypes are fitter. Trait divergence is easy to visualize when selection is disruptive. Stabilizing selection occurs when intermediate phenotypes are fittest.

These different modes of selection have implications for trait allometry and trait variation, particularly when dealing with sexually selected traits [43,69-71]. For example, directional selection is typically thought to be associated with positive allometry (where trait size increases faster than does body size, as frequently seen in sexually selected display traits), whereas stabilizing selection is likely to cause negative allometry (because intermediate values are favoured regardless of body size). Similarly, disruptive or directional selection are likely to lead, at least initially, to increased trait variation, whereas stabilizing selection should cause decreased variation.

direct link between genital form and fertilization success under sperm competition, and apparent cases have been documented in several other taxa (e.g. [21]).

\section{Genital divergence and mating systems}

A second prediction of the hypothesis that sexual selection drives genital divergence is that genital diversity should vary predictably among species according to mating system. The genitalia of species with polyandrous mating systems should be more divergent than those of monandrous species, in which females typically mate with only one male per reproductive attempt. Some of the earliest evidence that sexual selection influences genitalia was based on comparisons of genital complexity in species with different mating systems [1,22]. More recently, Arnqvist [23] compared male genital shape in monandrous and polyandrous species of beetles, flies, butterflies, moths and mayflies, using measures of geometric structural divergence and modern comparative methods. He found that genital divergence was about twice as great in groups in which females mate more than once, but that this difference did not occur in other morphological traits, such as wings or legs. This analysis strongly suggests that sexual selection occurring after intromission has driven insect genital divergence.

Not all across-species studies find evidence that sexual selection causes genital divergence. Similar to the insect aedeagus, the mammalian baculum or os penis is extremely species specific [24] but, although there is evidence for sexual selection causing baculum divergence in some groups, such as primates [24], this is not always the case [25-27]. Many of these studies are not exhaustive (e.g. [25]), and further investigation is needed to broaden the scope of these analyses in mammals, for example to explore potential associations between genital complexity and mating systems and/or copulatory behaviour. 


\section{Male and female coevolution}

Female reproductive organs are also frequently highly variable across closely related taxa [28] and, in one of the studies cited above, there was an interaction between female and male morphology that determined fertilization success [3]. Recent comparative studies have also begun to provide evidence of coevolution between male and female morphology, resulting from sexual conflict over mating rates $([29,30]$ but see e.g. [1]).

Arnqvist and Rowe [29] studied the coevolution of male and female morphologies used in the mating struggles of water striders. Results showed that the level of armament was closely correlated between the sexes, with males best adapted to grasp females in those species where females are best adapted to thwart them, and vice versa. Arnqvist and Rowe [30] also found a reduced frequency of superfluous mating among those species where female anticlasping devices are exaggerated relative to male grasping devices and, conversely, that relatively high rates of mating are more likely to occur where male armaments are exaggerated relative to those of females.

Water striders are a model system for studying sexual conflict, and there is currently far less information available concerning the relative costs and benefits of multiple mating for most other species. Experimental studies will thus be needed to complement comparative evidence of correlated evolution between male and female genitalia or related morphological traits in other species, because there are several potential explanations for correlated evolution between morphological traits. It is possible, for example, that correlations between male and female traits are caused by correlated evolution in response to more traditional forms of sexual selection (Box 3). A large-scale study by Eberhard [31] across insects and spiders found that genital divergence was not reduced in taxa where females are likely to be protected from unsolicited copulation attempts (i.e. where sexual conflict over copulation is unlikely). Thus, these results provide support for previous arguments that conflict over copulation has not influenced genital form widely $[1,5,6]$. However, because the reproductive interests of the sexes are rarely identical ([1] and see [32-34]), it is possible that other reproductive conflicts (e.g. over fertilization rather than mating per se) could still influence genital evolution [31].

\section{Female fitness costs associated with male genitalia}

A role for sexual conflict in genital evolution is also suggested by recent evidence for male genitalia sometimes causing injury and/or imposing other fitness-related costs to females. From a theoretical perspective, it has been argued that males can gain fertilization benefits by harming their mates, because if damage is cumulative over successive copulations, then females should be less likely to remate with competing males [35]. Alternatively or additionally, females might increase investment in current reproduction, thereby maximizing the reproductive success of their most recent mate, if they perceive genital damage as a threat to their future survival [36-38]. As yet, there is relatively little experimental support for either of these hypotheses [37,38]. The question of whether male genitalia are specifically adapted to cause damage to the female reproductive tract, or that damage occurs as a pleiotropic effect (of male adaptations for sperm competition for example) therefore remain matters of debate [26,37,38].

Nonetheless, there is evidence that reproductive costs for females can result from the action of male genitalia during copulation. For example, Crudgington and SivaJothy [36] describe how the intromittent organ of the male bean weevil Callosobruchus maculatus has sclerotized spines that are everted during copulation, apparently causing damage within the genital tract of the female. The longevity costs that arise from such damage are apparently not trivial, because multiply mated $C$. maculatus females had significantly reduced longevity compared with those that mated singly, although the fitness consequences of this are unclear. Male-inflicted damage could partly explain why female $C$. maculatus repeatedly kick their mates, which shortens the duration of copulation and reduces damage to their genital tracts. Similar results were found in the fly Sepsis cynipsea $[37,39,40]$. In this case, it appears male fitness is not enhanced by damaging females, and the selective advantage(s) for male malevolence remain unclear [37]. Further direct evidence of damage caused to females by male genitalia has also been reported in an invertebrate with a bizarre mode of copulation known as 'traumatic insemination' [41]. Male bed bugs Cimex lectularis use their external genitalia to pierce the abdominal wall of the female and inseminate directly into her body cavity. Females are apparently unable to resist repeated copulations, in spite of the associated costs of multiple mating, which reduce their longevity. In this group, the hypodermic aedeagus provides males with an alternative route to the eggs, but there is little genital divergence across bed bug species (W.G. Eberhard and M. Siva-Jothy, pers. commun.), again suggesting sexual conflict over fertilization plays little role in genital differentiation.

Whether penile spines found in vertebrates such as mammals cause injury to the female reproductive tract is uncertain, although comparative evidence for shorter estrus duration in primates with relatively well developed spines suggests that this is worthy of more direct investigation [26]. Injury and associated reduced longevity are not the only potential costs to females associated with stimulation by male genitalia. If, for example, females are less likely to remate as a consequence of increased copulatory stimulation or minor injury, they might also lose out on other benefits of polyandry [35,42]. However, potential long-term costs to females of remating below their optimal level are likely to be even more difficult to detect and quantify than are direct costs associated with harm or injury when mating above their optimal level, particularly among vertebrates.

\section{Genital allometry and variation}

The male-female interactions discussed above also imply there might be no single fitness peak for the size and/or shape of intromittent organs and, additionally, that males might be selected to fit the average female unless size assortative mating occurs [43]. If so, genital allometry 
should be negative (Box 3). In the most comprehensive analysis of genital size relative to body size, negative allometry was generally detected [43]. Although this study was criticized on methodological grounds [44], re-analysis did little to change the initial conclusions [45]. Negative genital allometry has subsequently been reported in other species [46], but there are cases of positive genital allometry $[47,48]$. Nevertheless, if the intromittent organ is generally under stabilizing selection, as appears to be the case [43], it raises the interesting question of how divergence is initiated. There are also few data currently available about how female genital tracts scale with body size. Without this information, it is difficult to evaluate the validity of the one-size-fits-all hypothesis, but available evidence is consistent with this idea, because the female genital tracts of species studied are negatively allometric $[43,49]$. Additionally, if female genital size has a singlepeaked distribution (e.g. leptokurtic or normal), male genital allometry might be negative regardless of how female genitalia scale.

The variability of genital traits has also been considered as evidence for sexual selection acting on them. Again, unless there is size assortative mating, it has been suggested that genitalia should have low coefficients of variation (CV) compared with nongenital morphology, reflecting the stronger stabilizing selection that they are under [43], and there is some evidence that genitalia have low CV [50]. However, in many instances, general morphological and genital traits display similar levels of variation [11,43,51]. House and Simmons [11] suggest that the low variation in genital size is the result of a balance between sexual and natural selection. Part of the problem is that there is no general theory suggesting what the patterns of variation should be, and potential pitfalls in comparing CV have largely been forgotten [52]. In particular, to perform unbiased CV comparisons, traits should be of equal complexity (or at the same structural level), which is not always the case when comparing genitalia and other characters. Additionally, CV differences (or similarity) might arise because of differences in the slope or dispersion of the data, and most studies typically do not distinguish between these distributional features [43].

\section{Concluding remarks}

Although current evidence is strongly in favour of sexual selection as the primary force driving genital diversity, it is less clear which sexual selection mechanisms are responsible. Four main sexual selection mechanisms have been implicated in genital evolution: sperm competition, good genes female choice, Fisherian female choice and sexual conflict (Box 2). Eberhard has strongly championed Fisherian selection $[1,5,6]$. However, there is considerable debate regarding the efficacy of runaway selection to influence evolutionary equilibria (Box 3). Eberhard also suggested that good genes sexual selection would be unlikely to drive genital divergence, because male genitalia are unlikely to reflect parasite resistance or general vigor. They are also frequently not size dependent or very variable (intraspecifically) and, hence, it seems that they are unlikely to be reliable indicator traits $[4,6]$. However, recent work with waterstriders shows that genital traits can be condition dependent [51], therefore conforming to the most recent good genes models of sexual selection [53] (and to expectations under sexual conflict), although they show less condition dependence than do other traits [54]. Additionally, in at least one study [3], copulatory courtship failed to explain variation in fertilization success, suggesting purely Fisherian effects were not operating. However, other measures of female preference for superior stimulators, such as variation in investment per offspring [55], were not investigated. More recently, Eberhard and co-workers have also argued that sexual conflict is unlikely to be the primary selective pressure causing genital divergence, in part, because of the negative allometry between genital and body size frequently reported $[4,43]$. However, because relatively little is known about allometric relationships generated under sexual conflict, it might be unsafe to conclude that allometry can always distinguish the selection mechanisms involved in genital evolution. More recent work by Eberhard has demonstrated that sexual conflict over mating is unlikely to have driven genital divergence, but other forms of conflict, for example over fertilization, cannot currently be rejected [31]. The evolution of traumatic insemination [41] seems to be one example of sexual conflict driving genital evolution. If so, explaining why there has been relatively little genital divergence in this group is of considerable interest.

In light of the unresolved issues discussed above, current arguments in favour of excluding sexual conflict as a potential selection pressure in genital evolution are perhaps premature [20,32]. Although the rapid diversification of genitalia is entirely consistent with sexual selection via a runaway process (but see Box 3), arms races generated by sexual conflict can also inevitably lead to trait divergence [33]. As noted by Alexander et al. [32] and others [1,20], elements of conflict and cooperation must exist in reproduction, so benefits and costs must therefore also exist, and it is measuring the balance between the two that is of most interest.

Distinguishing between alternative models of genital evolution by sexual selection is likely to prove difficult $[4,20,32]$, because each predicts fertilization successgenital form associations and, to some degree, good fit between male and female genitals. Although challenging, attempting to ascribe the relative strength of these processes is not beyond reach, and the powerful tool of laboratory evolution, so helpful in unraveling probable sexual selection mechanisms in other contexts [56-59], has yet to be brought to bear on this problem. Finally, we emphasize that these mechanisms need not be mutually exclusive and could potentially operate simultaneously in various combinations.

In conclusion, as championed by Eberhard [1], results of recent studies show that sexual selection is clearly a potent force in the evolution of genital diversity. As with other sexually selected traits, a key challenge for future studies will be to assess the relative importance of different sexual selection mechanisms in driving genital evolution. If Eberhard's belief $[1,5,6,31]$ that sexual conflict has had little widespread influence on genital evolution proves correct, this would be both a surprising and a significant 
finding, given the profound effects of evolutionary conflict at most other levels of selection [60]. The challenge of unraveling genital evolution thus offers an important step towards resolving current debate concerning the relative significance of sexual conflict and indirect benefits of mate choice in sexual selection.

\section{Acknowledgements}

We thank Oliver Martin, Helen Crudgington, Klaus Reinhold, Joe Tomkins, Brian Preston, Ted Morrow, Brett Holland, Matt Gage, an anonymous referee and especially Bill Eberhard for extremely helpful comments about the article. Bill also pointed out the potential paradox between the importance of conflict in sexual selection and other levels of selection. We also thank the Swiss Entomological Society for permission to reproduce the genitalia images used in Figure 1 and SNF and the Forschungskommission of the University of Zürich for financial support.

\section{References}

1 Eberhard, W.G. (1985) Sexual Selection and Animal Genitalia, Harvard University Press

2 Edwards, R. (1993) Entomological and mammalogical perspectives on genital differentiation. Trends Ecol. Evol. 8, 406-409

3 Arnqvist, G. and Danielsson, I. (1999) Copulatory behavior, genital morphology and male fertilization success in water striders. Evolution $53,147-156$

4 Eberhard, W.G. (2001) Species-specific genitalic copulatory courtship in sepsid flies (Diptera: Sepsidae, Microsepsis) and theories of genitalic evolution. Evolution 55, 93-102

5 Eberhard, W.G. (1998) Female roles in sperm competition. In Sperm Competition and Sexual Selection (Birkhead, T.R. and Miller, A.P., eds), pp. 91-118, Academic Press

6 Eberhard, W.G. (1997) Sexual selection by cryptic female choice in insects and arachnids. In The Evolution of Mating Systems in Insects and Arachnids (Choe, J.C. and Crespi, B.J., eds), pp. 32-57, Cambridge University Press

7 Eberhard, W.G. (1996) Female Control: Sexual Selection by Cryptic Female Choice, Princeton University Press

8 Parker, G.A. (1984) Sperm competition and the evolution of animal mating strategies. In Sperm Competition and the Evolution of Animal Mating Systems (Smith, R.L., ed.), pp. 1-60, Academic Press

9 Otronen, M. and Siva-Jothy, M.T. (1991) The effects of postcopulatory male behaviour on ejaculate distribution within the female sperm storage organs of the fly, Dryomyza anilis (Diptera: Dryomyzidae). Behav. Ecol. Sociobiol. 29, 33-37

10 Danielsson, I. and Askenmo, C. (1999) Male genital traits and mating interval affect male fertilization success in the water strider Gerris lacustris. Behav. Ecol. Sociobiol. 46, 149-156

11 House, C.M. and Simmons, L.W. (2002) Genital morphology and fertilization success in the dung beetle Onthophagus taurus: an example of sexually selected male genitalia. Proc. R. Soc. Lond. Ser. B $270,447-455$

12 Otronen, M. (1998) Male asymmetry and postcopulatory sexual selection in the fly Dryomyza anilis. Behav. Ecol. Sociobiol. 42, $185-191$

13 Rodriguez, V. et al. Tortoise beetle genitalia and demonstrations of sexually selected advantage for flagellum length in Chelymorpha alternans (Chrysomelidae, Cassidini, Stolaini). In New Developments on the Biology of Chrysomelidae (Jolivet, P. et al., eds), SPB Academic Publishing (in press)

14 Tadler, A. (1999) Selection of a conspicuous male genitalic trait in the seedbug Lygaeus simulans. Proc. R. Soc. Lond. Ser. B 266, 1773-1777

15 Tadler, A. et al. (1999) Selection of male traits during and after copulation in the seedbug Lygaeus simulans (Heteroptera Lygaeidae). Biol. J. Linn. Soc. Lond. 68, 471-483

16 Cordoba-Aguilar, A. (1999) Male copulatory sensory stimulation induces female ejection of rival sperm in a damselfly. Proc. $R$. Soc. Lond. Ser. B 266, 779-784

17 Cordoba-Aguilar, A. (2002) Sensory trap as the mechanism of sexual selection in a damselfly genitalic trait (Insecta: Calopterygidae). Am. Nat. 160, 594-601
18 Waage, J.K. (1979) Dual function of the damselfly penis: sperm removal and transfer. Science 203, 916-918

19 Robinson, J.V. and Novak, K.L. (1997) The relationship between mating system and penis morphology in ischnuran damselflies (Odonata: Coenagrionidae). Biol. J. Linn. Soc. Lond. 60, 187-200

20 Simmons, L.W. (2001) Sperm Competition and its Evolutionary Consequences in the Insects, Princeton University Press

21 Haubruge, E. et al. (1999) Fertilization by proxy: rival sperm removal and translocation in a beetle. Proc. R. Soc. Lond. Ser. B 266, 1183-1187

22 Dixson, A.F. (1987) Observations on the evolution of the genitalia and copulatory behavior in male primates. J. Zool. 213, 423-443

23 Arnqvist, G. (1998) Comparative evidence for the evolution of genitalia by sexual selection. Nature 393, 784-786

24 Dixson, A.F. (1998) Primate Sexuality: Comparative Studies of the Prosimians, Monkeys, Apes and Human Beings, Oxford University Press

25 Hosken, D.J. et al. (2001) Is the bat os penis sexually selected? Behav. Ecol. Sociobiol. 50, 450-460

26 Stockley, P. (2002) Sperm competition risk and male genital anatomy: comparative evidence for reduced duration of female receptivity in primates with penile spines. Evol. Ecol. 16, 123-137

27 Larviere, S. and Ferguson, S.H. (2002) On the evolution of the mammalian baculum: vaginal friction, prolonged intromission or induced ovulation? Mamm. Rev. 32, 283-294

28 Pitnick, S. et al. (1999) Evolution of multiple kinds of female spermstorage organs in Drosophila. Evolution 53, 1804-1822

29 Arnqvist, G. and Rowe, L. (2002) Correlated evolution of male and female morphologies in water striders. Evolution 56, 936-947

30 Arnqvist, G. and Rowe, L. (2002) Antagonistic coevolution between the sexes in a group of insects. Nature 415, 787-789

31 Eberhard, W.G. Male-female conflict and genitalia: failure to confirm predictions in insects and spiders. Biol. Rev. (in press)

32 Alexander, R.D. et al. (1997), Evolutionary perspectives on insect mating. In The Evolution of Mating Systems in Insects and Arachnids (Choe, J.C. and Crespi, B.J., eds), pp. 4-31, Cambridge University Press

33 Rice, W.R. and Holland, B. (1997) The enemies within: intergenomic conflict, interlocus contest evolution (ICE), and the intraspecific Red Queen. Behav. Ecol. Sociobiol. 41, 1-10

34 Parker, G.A. (1979) Sexual selection and sexual conflict. In Sexual Selection and Reproductive Competition in Insects (Blum, N.S. and Blum, N.A., eds), pp. 123-166, Academic Press

35 Johnstone, R.A. and Keller, L. (2000) How males can gain by harming their mates: sexual conflict, seminal toxins, and the cost of mating. Am. Nat. 156, 368-377

36 Crudgington, H.S. and Siva-Jothy, M.T. (2000) Genital damage, kicking and early death. Nature 407, 655-656

37 Hosken, D.J. et al. (2003) Sexual conflict in Sepsis cynipsea: female reluctance, fertility and mate choice. J. Evol. Biol. 16, 485-490

38 Morrow, E.H. et al. Adaptation versus pleiotropy: why do males harm their mates? Behav. Ecol. (in press)

39 Blanckenhorn, W.U. et al. (2002) The cost of copulating in the dung fly Sepsis cynipsea. Behav. Ecol. 13, 353-358

40 Martin, O.Y. et al. (2003) Male age, mating probability and mating costs in the fly Sepsis cynipsea. Evol. Ecol. Res. 5, 119-129

41 Stutt, A.D. and Siva-Jothy, M.T. (2001) Traumatic insemination and sexual conflict in the bed bug Cimex lectularius. Proc. Natl. Acad. Sci. U. S. A. $10,5683-5687$

42 Hosken, D.J. and Stockley, P. (2003) Benefits of polyandry: a life history perspective. J. Evol. Biol. 33, 173-194

43 Eberhard, W.G. et al. (1998) One size fits all? Relationships between the size and degree of variation in genitalia and other body parts in twenty species of insects and spiders. Evolution 52, 415-431

44 Green, A.J. (1999) Allometry of genitalia in insects and spiders: one size does not fit all. Evolution 53, 1621-1624

45 Eberhard, W.G. et al. (1999) Don't forget the biology: a reply to Green. Evolution 53, 1624-1627

46 Berstein, S. and Berstein, R. (2002) Allometry of male genitalia in a species of soldier beetle: support for the one-size-fits-all hypothesis. Evolution 56, 1707-1710

47 Kelly, C.D. et al. (2000) Geographical variation in the male intromittent organ of the Trinidadian guppy (Poecilia reticulata). Can. J. Zool. 78, 1674-1680 
48 Miller, E.H. and Burton, L.E. (2001) It's all relative: allometry and variation in the baculum (os penis) of the harp seal, Pagophilus groenlandicus (Carnivora: Phocidea). Biol. J. Linn. Soc. Lond. 72, 345-355

49 Gage, M.J.G. (1998) Influences of sex, size, and asymmetry on ejaculate expenditure in a moth. Behav. Ecol. 9, 592-597

50 Uhl, G. and Vollrath, F. (2000) Extreme body size variability in the golden silk spider does not extend to genitalia. J. Zool. 251, 7-14

51 Arnqvist, G. and Thornhill, R. (1998) Evolution of animal genitalia: patterns of phenotypic and genotypic variation and condition dependence of genital and non-genital morphology in water strider (Heteroptera: Gerridae: Insecta). Genet. Res. 71, 193-212

52 Lande, R. (1977) On comparing coefficients of variation. Syst. Zool. 26, 214-217

53 Rowe, L. and Houle, D. (1996) The lek paradox and the capture of genetic variance by condition dependent traits. Proc. R. Soc. Lond. Ser. $B$ 263, 1415-1421

54 Arnqvist, G. et al. (1997) Evolution of animal genitalia: morphological correlates of fitness components in a water strider. J. Evol. Biol. 10, $613-640$

55 Cunningham, E.J.A. and Russell, A.F. (2000) Egg investment is influenced by male attractiveness in the mallard. Nature 404, 74-77

56 Rice, W.R. (1996) Sexually antagonistic male adaptation triggered by experimental arrest of female evolution. Nature 381, 232-234

57 Miller, G.T. and Pitnick, S. (2002) Female sperm co-evolution in Drosophila. Science 298, 1230-1233

58 Gage, M.J.G. and Morrow, E.H. (2003) Experimental evidence for the evolution of numerous, tiny sperm via sperm competition. Curr. Biol. 13, 754-757

59 Martin, O.Y. and Hosken, D.J. (2003) The evolution of reproductive isolation through sexual conflict. Nature 423, 979-982
60 Keller, L. ed. (1999) Levels of Selection in Evolution, Princeton University Press

61 Mayr, E. (1963) Animal Species and Evolution, Harvard University Press

62 Cordero, C. and Eberhard, W.G. (2003) Female choice of sexually antagonistic adaptations. A critical review of some current research. J. Evol. Biol. 16, 1-6

63 Cameron, E. et al. (2003) Sexual conflict and indirect benefits. J. Evol. Biol. 16, 1055-1060

64 Kirkpatrick, M. (1985) Evolution of female choice and male parental investment in polygynous species: the demise of the sexy son. Am. Nat. $125,788-810$

65 Gavrilets, S. et al. (2001) The evolution of female mate choice by sexual conflict. Proc. R. Soc. Lond. Ser. B 268, 531-539

66 Shuster, S.M. and Wade, M.J. (2003) Mating Systems and Strategies, Princeton University Press

67 Lessells, C.M. (1999) Sexual conflict in animals. In Levels of Selection in Evolution (Keller, L., ed.), pp. 75-99, Princeton University Press

68 Ryan, M.J. (1990) Sexual selection, sensory systems and sensory exploitation. Oxf. Surv. Evol. Biol 7, 156-195

69 Petrie, M. (1988) Intraspecific variation in structures that display competitive ability: large males invest relatively more. Anim. Behav. $36,1174-1179$

70 Petrie, M. (1992) Are all secondary sexual display structures positively allometric and, if so, why? Anim. Behav. 43, 173-175

71 Simmons, L.W. and Tomkins, J.L. (1996) Sexual selection and allometry of earwig forceps. Evol. Ecol. 10, 97-104

72 Bächli, G. and Vilela, C.R. (2002) Six new species of Drosophila (Diptera Drosophilidae) from Parque Nacional Henri Pittier, Venezuela. Mitt. Schweiz. Ent. Ges. 75, 223-243

73 Vilela, C.R. and Bächli, G. (2002) Two new North American Drosophila species (Diptera Drosophilidae). Mitt. Schweiz. Ent. Ges. 75, 211-221 